MATEC Web of Conferences 44, 02091 (2016)

DOI: $10.1051 /$ matecconf/20164402091

(C) Owned by the authors, published by EDP Sciences, 2016

\title{
Variation function fitting method based on particle swarm optimization
}

\author{
Ping Duan ${ }^{1}$, Jia Li ${ }^{1 a}$, Hai Yang $\mathrm{Lv}^{2,3,4}$ \\ ${ }^{1}$ School of Tourism and Geographical Sciences of Yunnan Normal University, Kunming, 650050, China \\ ${ }^{2}$ Key Laboratory of Virtual Geographic Environment (Nanjing Normal University), Ministry of Education, Nanjing, 210023, China \\ ${ }^{3}$ State Key Laboratory Cultivation Base of Geographical Environment Evolution (Jiangsu Province), Nanjing, 210023, China \\ ${ }^{4}$ Jiangsu Center for Collaborative Innovation in Geographical Information Resource Development and Application, Nanjing, 210023, China
}

\begin{abstract}
In the Kriging interpolation method, different theory models of variation function are selected and fitted. There are many common variation function models, such as spherical model, index model, Gaussian model and so on. As these variation function models are non-linear, non-linear model are converted to linear model when these variation function models are solved. Different variation function models with different conversion methods are lack of generality in the process of Kriging interpolation. Particle swarm optimization algorithm with the advantages of global optimal solution can be directly used to solve non-linear fitting equation. In this paper, variation function model based on particle swarm optimization algorithm is fitted. Experiment shows that it is appropriate for fitting variable function based particle swarm optimization algorithm.
\end{abstract}

\section{Introduction}

Kriging interpolation is founded by South African geological engineer Krige. And then, Kriging interpolation theory is improved by French statisticians Matheron [1]. Kriging interpolation method as one of the common spatial interpolation has been widely used in geology, atmosphere, soil and remote sensing. Like most of the spatial interpolation method, Kriging interpolation is also based on the principle of spatial correlation. The closer distance between space objects, the greater the similarity of spatial properties. The basic principle of Kriging interpolation is that real variation function values of sampled data are firstly calculated in interpolation process. And then, theory model of variation function is constructed. Weights and Lagrange coefficient between reference points and interpolation point are calculated based on variation function model. Finally, attribute values of interpolation point are calculated by solving linear combination equations.

Variation function model is the basis of Kriging interpolation method. It is a tool to reflect the rule of regionalized variable and ultimately determines the authenticity and effectiveness of prediction results [2]. There are many common variation function models, such as linear sill model, spherical model, exponential model, Gaussian model, logarithmic model and so on[3]. These models can be divided into non-linear and linear curve fitting model. For linear curve model, variation function can be directly fitted by least squares. Compared with linear curve model, non-linear model are converted to linear model when these variation function models are solved. The difficulty of conversion increases the complexity of algorithm.

With the development of non-linear theory in recent years, non-linear curve for solving need not be converted linear curve. It can be directly solved by intelligent algorithm. Particle swarm optimization (PSO) algorithm as a kind of typical intelligent algorithm has the advantages of global optimal solution. It can not only directly solve non-linear fitting equation but also solve variation function fitting equation. In this paper, particle swarm optimization algorithm is used to fit variation function model. In order to verify the variation function theory model based on particle swarm fitting method, iron ore grade data from some region in china is tested. The fitting result of PSO algorithm is the same with the weighted least squares algorithm. As can be seen from the experimental results, the weighted least squares algorithm can be replaced by PSO algorithm. It can avoid the conversion from non-linear to linear.

\section{Kriging interpolation model and method}

Kriging interpolation is an optimal linear unbiased estimation method. Kriging interpolation similar with inverse distance weighted is based on sampling points around unknown points to calculate the value of unknown points. With the development of Kriging interpolation, there are many kriging interpolation methods. The basic principle of these methods are the same, so ordinary Kriging interpolation model is researched in this paper.

\footnotetext{
${ }^{\mathrm{a}}$ Corresponding author: 1ijia8425@163.com
} 


\subsection{Kriging interpolation model}

Supposed regionalized variable $Z(\mathrm{x})$ meet second order stationarity conditions. There are $n$ reference points around the interpolation point $\mathrm{x}$ with attribute value $\mathrm{Z}\left(x_{i}\right)$. The Kriging interpolation model is [4]:

$$
Z^{\prime}(\mathrm{x})=\sum_{i=1}^{n} \lambda_{i} Z\left(\mathrm{x}_{i}\right)
$$

Here, $\lambda$ is weight. Formula (1) need to meet the following conditions:

(1) Condition of unbiasedness:

$$
\sum_{i=1}^{n} \lambda_{i}=1
$$

(2) Condition of minimum variance:

$$
\sigma_{E}^{2}=E\left[\mathrm{Z}^{\prime}(\mathrm{x})-\mathrm{Z}(\mathrm{x})\right]^{2}=E\left[\mathrm{Z}(\mathrm{x})-\sum_{i=1}^{n} \lambda_{i} \mathrm{Z}\left(\mathrm{x}_{\mathrm{i}}\right)\right]^{2}=\min
$$

In order to minimize the estimated value of variance, according to the principle of Lagrange multipliers, let be:

$$
F=\sigma_{E}^{2}-2 \mu\left(\sum_{i=1}^{n} \lambda_{i}-1\right)
$$

Here, $\mu$ is Lagrange multipliers.

Partial derivative of $F$ is calculated and let be the value of partial derivative is zero. Equation set of Kriging are as follows:

$$
\left\{\begin{array}{c}
\frac{\partial F}{\partial \lambda_{i}}=2 \sum_{j=1}^{n} \lambda_{j} c\left(\mathrm{x}_{i}, \mathrm{x}_{j}\right)-2 c\left(\mathrm{x}_{i}, \mathrm{x}\right)-2 \mu=0 \\
\frac{\partial F}{\partial \mu}=-2\left(\sum_{i=1}^{n} \lambda_{i}-1\right)=0
\end{array}\right.
$$

Here, $c$ is covariance. According to the relationship of covariance and variation function,

$$
\gamma(h)=c(0)-c(h)
$$

Here, $h$ is the distance between point pairs. $\gamma$ is the value of variation function.

Finally, the interpolation model can be expressed as follows:

$$
\left[\begin{array}{cccc}
\gamma_{11} & \cdots & \gamma_{1 n} & 1 \\
\vdots & \ddots & \vdots & \vdots \\
\gamma_{n 1} & \cdots & \gamma_{n n} & 1 \\
1 & \cdots & 1 & 0
\end{array}\right] \cdot\left[\begin{array}{c}
\lambda_{1} \\
\vdots \\
\lambda_{n} \\
\mu
\end{array}\right]=\left[\begin{array}{c}
\gamma_{10} \\
\vdots \\
\gamma_{n 0} \\
1
\end{array}\right]
$$

\subsection{Kriging interpolation method}

Concrete steps of Kriging interpolation are as follows [5]:

(1) Normal distribution of sampling data is determined. If sampling data not meet normal distribution, sampling data need to convert approximate normal distribution.

(2) Variation function values of sampling data are calculated. If the size of point pairs is large, variation function values with different groups are calculated.

(3) Theory model of variation function is selected and variation function curve is fitted.

(4) Weight matrix of interpolation point is calculated according to the variation function curve.

(5) The attribute value of unknown point are calculated according to linear combination of weight matrix.
In the process of Kriging interpolation, step (3) is the key step. Because the variation function curve affects the weight matrix of the interpolation point. In this paper, the particle swarm optimization algorithm is used to fit variation function curve.

\section{Theory model of variation function based on particle swarm optimization algorithm}

Particle swarm optimization algorithm (PSO for short) is proposed by Kennedy and Eberhart in 1995. It is a kind of swarm intelligence algorithm that simulated the search process of birds group [6]. If there is only one piece of food in some region, the most simple and effective strategy to find the food is to search the nearest area around birds.

In PSO algorithm, birds as a particle in the space, has their own position. It can be expressed by $\left(x_{i}, v_{i}\right.$, pbest $\left._{i}\right) . x_{i}$ is the current position of the particle. $v_{i}$ is the current speed of the particle. pbest $t_{i}$ is the best current position that has been searched by particle. An objective function (denoted by Fitness) is given to each particle's position. The fitness of each particle can be evaluated by Fitness function. The value of Fitness function as evaluation criterion is calculated. It can update the current optimal value of the particle, denoted by pbest $t_{i}$.

In the algorithm implementation process, some initial values are given, such as the particle position, the speed of the particles, inertia weigh of the particle $w_{i}$, randomly learning factor of the particle $c_{1}$ and $c_{2}$. Optimal solution of current particle pbest $t_{i}$ and all particles are solved by iterative search of each particle. gbest $_{i}$ is the global extremum. pbest $_{i}$ and gbest $_{i}$ are velocity parameters of next updating. By $n$ iterations, it finally can get a satisfactory result.

Mathematical expressions of update speed $v$ and position $x$ of particle are as follows:

$$
\begin{gathered}
v_{i, j}^{k+1}=w_{i} v_{i, j}^{k}+c_{1} \cdot \operatorname{rand}_{1} \cdot\left(\text { pbest }_{i, j}^{k}-x_{i, j}^{k}\right) \\
+c_{2} \cdot \operatorname{rand}_{2} \cdot\left(\operatorname{gbest}_{i, j}^{k}-x_{i, j}^{k}\right) \\
x_{i, j}^{k+1}=x_{i, j}^{k}+v_{i, j}^{k+1}
\end{gathered}
$$

Here, $(i, j)$ is the index of the current particle. The superscript $k$ is the $k$-th iteration.

The algorithm of Particle Swarm Optimization is as follows:

Table 1. The algorithm process of PSO

The algorithm of PSO

Initialize the related parameters and scope of particles

For $i=1$ : times of iterations

For $j=1:$ sum of particles

To update the current particle velocity;

To update the current particle location;

Local optimal fitness function calculation;

Global optimal fitness function calculation;

End

End

Output optimal particle location (optimal parameters) 
The flow chart is as follows :

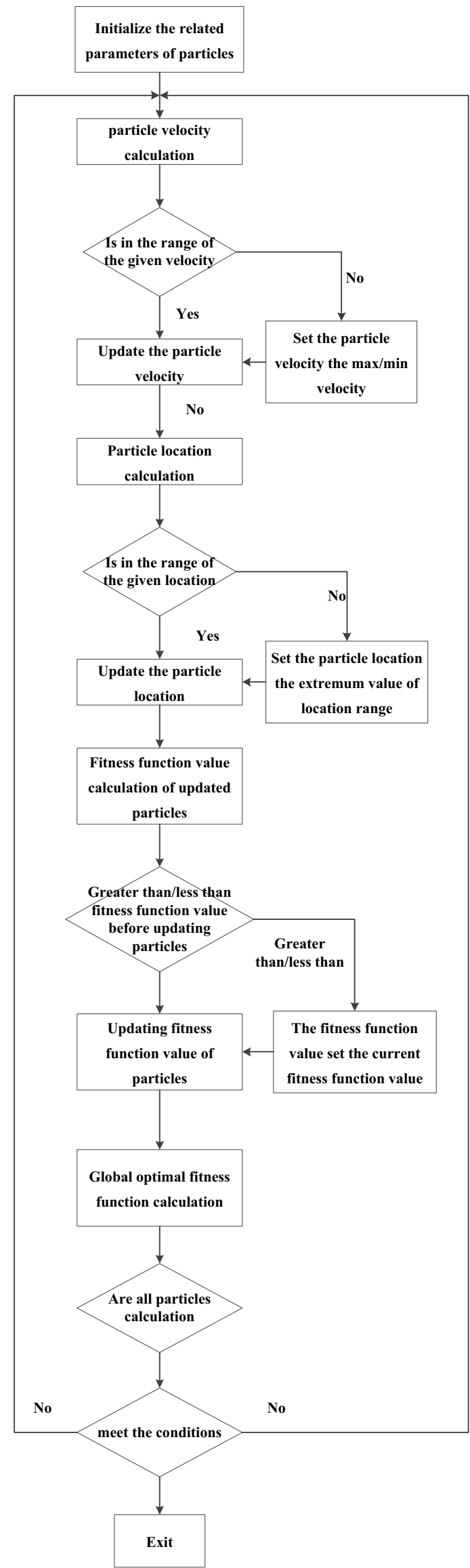

The purpose of variation function model fitting is to minimize error of curve fitting. Error corresponds to the Fitness function in PSO algorithm when curve is fitted. The mathematical expression is:

$$
\text { Fitness }=\sum_{j=1}^{n}\left[\gamma\left(h_{j}\right)-\gamma^{\prime}\left(h_{j}\right)\right]^{2}
$$

Here, $h_{j}$ is separation distance of variation function. $\gamma\left(h_{j}\right)$ is an actual value of variation function of separation distance $h_{j} . \gamma^{\prime}\left(h_{j}\right)$ is the theory model value of current variation function.

In the actual process of variation function fitting, weight value is large when the distance of two points are closer in variation function diagram and vice versa. Therefore, with distance as the weight, the definition of Fitness function is:

$$
\text { Fitness }=\sum_{j=1}^{n} w_{j} \cdot\left[\gamma\left(h_{j}\right)-\gamma^{\prime}\left(h_{j}\right)\right]^{2}
$$

Here,

$$
\begin{gathered}
w_{j}=w_{i} / \sum_{i=1}^{n} w_{i} \\
w_{i}=h_{j} / h_{\text {all }}
\end{gathered}
$$

Here, $h_{\text {all }}$ is the sum of all the separation distance $h_{j}$.

The number of unknown in variation function theory model and particle in PSO algorithm are the same. The position of particle represents the unknown in variation function theory model. The particle position of minimum Fitness function value is searched by PSO algorithm, which is the expression of curve fitting.

\section{The experiment and conclusion}

In order to verify the variation function theory model based on particle swarm fitting method, iron ore grade data from some region in china is tested. Iron ore grade in 3D space presents anisotropic characteristics. Variation function values of three orientations are calculated and fitted by spherical model.

Supposed the range of three particles' positions is [0, 20]. The number of particle is 15 . The iteration times are 30. Spherical theory model of variation function is fitted and solved by PSO algorithm. Fitting parameters of three orientations are shown in table 2.

Table 2. Fitting parameters of spherical model of variation function on three orientations

\begin{tabular}{|c|c|c|c|}
\hline Orientation & Nugget (c0) & High arch (c) & Range (a) \\
\hline $\mathrm{x}$ & $1.3753 \mathrm{E}-02$ & $2.5131 \mathrm{E}-02$ & $5.4387 \mathrm{E}+02$ \\
\hline $\mathrm{y}$ & $1.2412 \mathrm{E}-02$ & $1.7804 \mathrm{E}-02$ & $4.4146 \mathrm{E}+02$ \\
\hline $\mathrm{z}$ & $2.2286 \mathrm{E}-04$ & $5.2774 \mathrm{E}-02$ & $5.2431 \mathrm{E}+02$ \\
\hline
\end{tabular}

The fitting results of three orientations are shown in Figure 2 .

Figure 1. The flow chart of PSO algorithm 


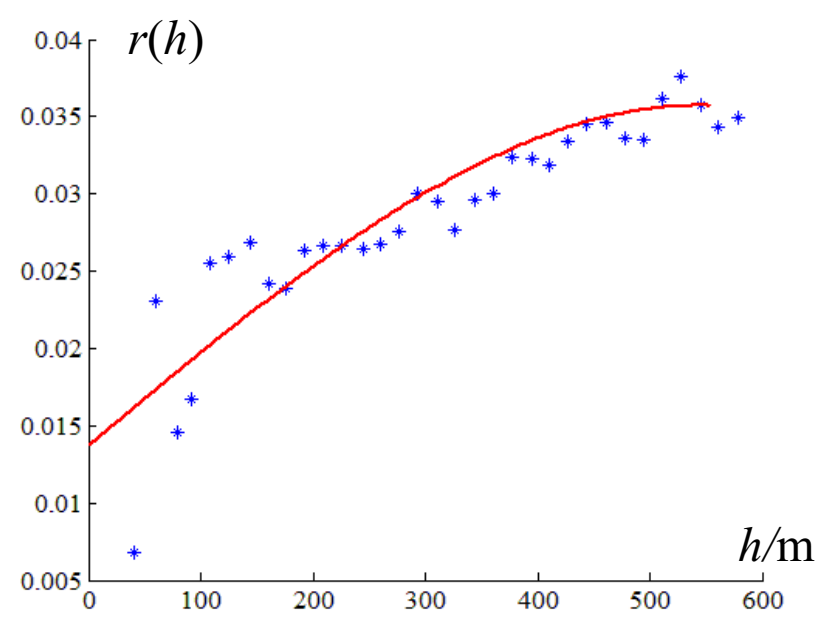

(a) Fitting curve of variation function on $x$ orientation

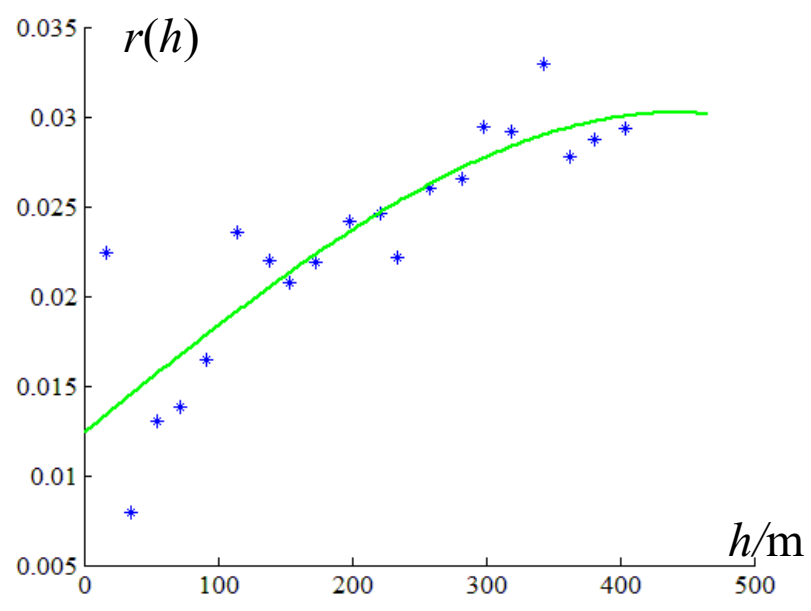

(b) Fitting curve of variation function on $y$ orientation

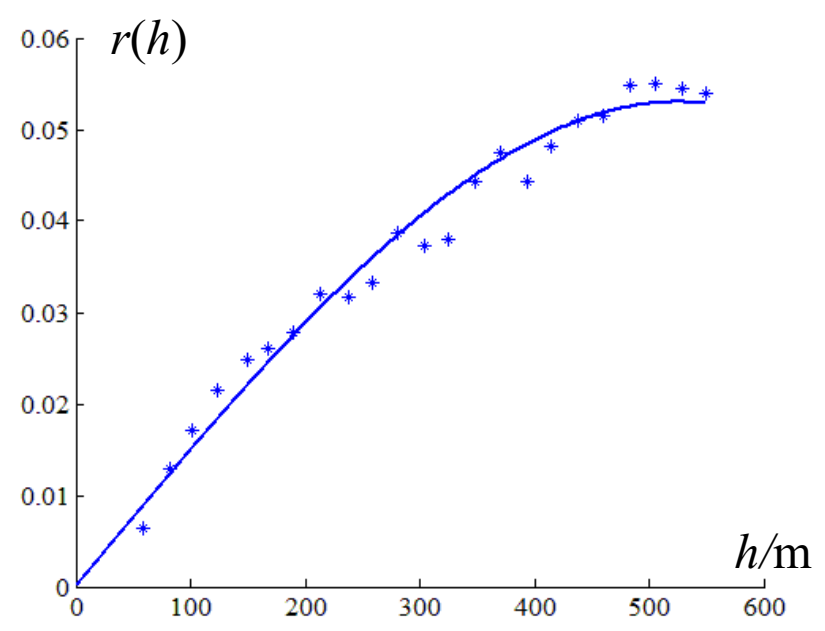

(c) Fitting curve of variation function on $z$ orientation

Figure 2. Fitting parameters of spherical model of variation function on three orientations

Distance weighted fitting is adopted by Fitness function of PSO algorithm. The fitting result of PSO algorithm is the same with the weighted least squares algorithm. As can be seen from the experimental results, the weighted least squares algorithm can be replaced by PSO algorithm. It can avoid the conversion from non- linear to linear. Therefore, PSO algorithm is an effective method for variation function fitting.

\section{Acknowledgements}

This work has been Supported by Youths Foundation of School of Tourism and Geographical Sciences of YunNan Normal University, Doctoral Fund of YunNan Normal University (No. 01300205020503113) and the National Natural ScienceFoundation of China (No.41271383).

\section{References}

1. G. Matheron, Principles of geostatistics. Economic Geology, 58, 8(1963)

2. Y. Jin, M. Pan, C. Ma, Principles and Validation of unified Approach for Nested Overlap of Geological Variable Variograms. Journal of China University of Mining \& Technolo, 39, 3(2010)

3. A. L. Liu, P. F. Wang, Y. Y. Ding, Introduction to Geostatistics, Science Press in China, (2012)

4. J. P. Yue, Z. K. Zhen, Application of Particle Swarm Optimization Based Kriging Interpolation Method in Regional Land Subsidence. Surveying and mapping, 3,(2012)

5. H. Q. Sun, Geostatistics and its application, China University of Mining and Technology press, (1990)

6. L. Q. Yao, M. Pan, Q. M. Cheng, Nested Overlap of Variograms in 3D Kriging, Earth Science-Journal of China University of Geosciences, 34,2(2009).

7. J. H. Qiao, M. Pan,Y.Jin, 3D modeling and Analysis of Natural Foundation Beating Capacity Based on Kriging Method, Acta Scientiarum Naturalium Universitatis Pekinensis, 47, 5 (2011)

8. X. C. Mao, F. Cao, Y. H. Zou, Y. Zhao, Construction and application of variation ellipsoid for analysis of mineralization structure, Journal of Central South University(Science and Technology), 45, 9(2014)

9. F. GAO, Research on Intelligent Particle Swarm Optimization Algorithm, Harbin Institute of Technology, (2008)

10. R. C. Eberhart, J. Kennedy, A New Optimizer Using Particle Swarm Theory. Proceedings of the Sixth International Symposium on Micro Machine and Human Science, Nagoya(1995)

11. J. Kennedy, R. C. Eberhart. Particle Swarm Optimization. Proceeding of IEEE International Conference on Neural Networks. Piscataway, (1995)

12. X. H. Shi, Y. C. Liang, H. P. Lee, C. Lu, Q. X. Wang, Particle swarm optimization-based algorithms for TSP and generalized TSP. Information Processing Letters, (2007) 